\title{
The hBracelet: a wearable haptic device for the distributed mechanotactile stimulation of the upper limb
}

This is the peer reviewed version of the following article:

Original:

Meli, L., Hussain, I., Aurilio, M., Malvezzi, M., O'Malley, M., Prattichizzo, D. (2018). The hBracelet: a wearable haptic device for the distributed mechanotactile stimulation of the upper limb. IEEE ROBOTICS AND AUTOMATION LETTERS, 3(3), 2198-2205 [10.1109/LRA.2018.2810958].

Availability:

This version is availablehttp://hdl.handle.net/11365/1036932

since 2019-04-09T19:15:15Z

Published:

DOI:10.1109/LRA.2018.2810958

Terms of use:

Open Access

The terms and conditions for the reuse of this version of the manuscript are specified in the publishing policy. Works made available under a Creative Commons license can be used according to the terms and conditions of said license.

For all terms of use and more information see the publisher's website.

(Article begins on next page) 


\title{
The hBracelet: a wearable haptic device for the distributed mechanotactile stimulation of the upper limb
}

\author{
Leonardo Meli $^{1}$, Irfan Hussain ${ }^{1}$, Mirko Aurilio ${ }^{1}$, Monica Malvezzi ${ }^{1}$, Marcia K. O’Malley ${ }^{3}$, and Domenico Prattichizzo ${ }^{1,2}$
}

\begin{abstract}
Haptic interfaces are mechatronic devices designed to render tactile sensations; although they are typically based on robotic manipulators external to the human body, recently, interesting wearable solutions have been presented. Towards a more realistic feeling of virtual and remote environment interactions, we propose a novel wearable skin stretch device for the upper limb called "hBracelet". It consists of two main parts coupled with a linear actuator. Each part contains two servo actuators that move a belt. The device is capable of providing distributed mechanotactile stimulation on the arm by controlling the tension and the distance of the two belts in contact with the skin. When the motors spin in opposite directions, the belt presses into the user's arm, while when they spin in the same direction, the belt applies a shear force to the skin. Moreover, the linear actuator exerts longitudinal cues on the arm by moving the two parts of the device. In this work we illustrate the mechanical structure, working principle, and control strategies of the proposed wearable haptic display. Furthermore, we present a qualitative experiment in a teleoperation scenario as a case study to demonstrate the effectiveness of the proposed haptic interface and to show how a human can take advantage of multiple haptic stimuli provided at the same time and on the same body area. The results show that the device is capable of successfully providing information about forces acting at the remote site, thus improving the overall telepresence.
\end{abstract}

\section{INTRODUCTION}

The complexity of the world around us is creating a demand for novel interfaces that will simplify and enhance the way we interact with the environment. The interaction that happens between the external environment and the user, mediated by a robotic manipulator, represents a typical teleoperation scenario, where the human user is the master and the artificial hand, manipulating external objects, is the slave [?]. In order to enable the user to have a more natural and realistic interaction during tele-operation tasks, it is important to provide the user with haptic sensations arising from such an interplay. In this respect, there is a variety of new wearable devices, called "wearables," that

This research has received funding from the European Union Seventh Framework Programme FP7/2007-2013 under grant agreement $n^{\circ} 601165$ of the project "WEARHAP" and from the European Union's Horizon 2020 Research and Innovation Programme under Grant Agreement $n^{\circ} 688857$ of the project "SoftPro".

${ }^{1}$ Dept. of Information Engineering, University of Siena, Via Roma 56, 53100 Siena, Italy. \{leonardo.meli, irfan.hussain, mirko.aurilio, monica.malvezzi, domenico.prattichizzo\}eunisi.it

${ }^{2}$ Dept. of Advanced Robotics, Istituto Italiano di Tecnologia, Via Morego 30, 16163 Genoa, Italy.

${ }^{3}$ Dept. of Mechanical Engineering, Rice University, 6100 Main Street, Houston, TX, USA. omalleymerice.edu

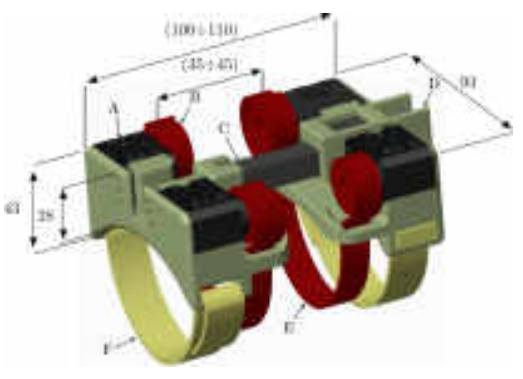

Fig. 1. The hBracelet. It consists of four servo motors and one linear actuator. The structural frame of the device is $3 \mathrm{D}$ printed

have been developed for this purpose. Wearables enable novel forms of communication, cooperation, and integration between humans and robots. Specifically, they enable the communication between the human wearer and the robotic device during the interaction with the environment they share. Different types of stimuli might be rendered on the human skin, such as information of pressure [?], and proprioceptive and directional cues [?], which are mainly related to skin stretch and deformation [?].

In this regard, we find cutaneous technologies very promising. Cutaneous cues are sensed by mechanoreceptors in the skin and they are useful to recognize the local properties of objects, e.g., shape, edges, embossings, and recessed features [?], [?]. The richness of information cutaneous receptors are able to detect, together with their broad distribution throughout the body, make the skin a perfect channel to communicate with the human user. Moreover, cutaneous haptic feedback represents an effective and elegant way to simplify the design of haptic interfaces: the low activation threshold of skin receptors enables the design of small, lightweight, and inexpensive devices [?], [?], [?]. Cutaneous feedback has been also proven to play a key role in enhancing the performance and effectiveness of teleoperation systems [?], [?], as well as for the intuitive control of a prosthetic limb for transradial amputees [?].

Although there is a growing interest in wearable haptic displays, most of these are based on solely vibrotactile signals, or have limited force feedback modalities. In this work we present the $\mathrm{hBracelet}$ that is able to provide a multimodal mechanotactile stimulation at the same time and on the same body area, i.e., the user arm, through pressure and stretch cues related to normal, tangential, and longitudinal forces (see Fig. 1). 


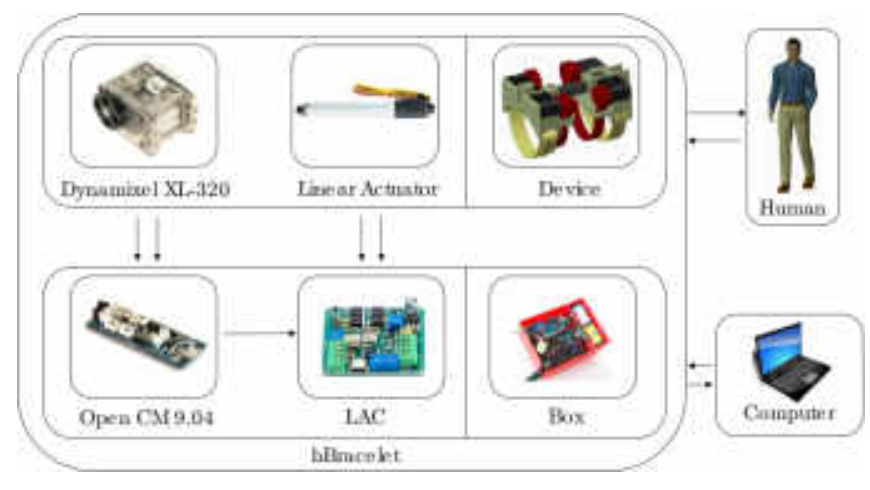

Fig. 2. System block diagram of the complete system. The actuators of the hBracelet are connected to their controllers which in turn are connected to the computer.

As a case study, we exploited the hBracelet in a telemanipulation scenario. The results show that the device is capable of informing the operator about different actions performed at the remote environment and allows her/him to successfully discern multiple haptic signals yielded on the same body area.

The rest of the paper is organized as it follows: Sec. II reports details on the device design and realization; Sec. III presents the mathematical formulation of the device; Sec. IV presents an application of the hBracelet in a teleoperation scenario and Sec. V the results and discussion of this qualitative experiment; finally, Sec. VI provides concluding remarks and perspectives of the work.

\section{THE HBRACELET}

To provide mechanotactile stimulation to the human upper limb we have designed the hBracelet, a wearable haptic interface whose CAD model is depicted in Fig. 1.

\section{A. Hardware description}

The hBracelet is composed of two main parts coupled with a Micro Linear Actuator L12-P (Actuonix, Canada) (C). The structural frame (D) of the device is symmetrical and 3D printed with polymeric ABS (Acrylonitrile Butadiene Styrene, ABSPlus, Stratasys, USA). Both parts of the device are composed of two Dynamixel XL-320 (Robotis, South Korea) actuators (A), two pulleys (B), a 3D printed thermoplastic polyurethane (Lulzbot, USA) belt (E), and a Velcro strap (F) for size adjusting. The distance between the front and rear belts is set following the perception guidelines present in the literature to let the subject distinguish between two independent haptic cues [?]. The hBracelet has a dimension of $(9.3 \times 11.0 \times 2.8) \mathrm{cm}$ and weighs $3.6 \mathrm{~g}$. For a complete description of the motors and the linear actuator, the reader is referred to [?] and [?].

\section{B. Control}

Fig. 2 shows the block diagram of the complete system. An OpenCM9.04-C controller is used to drive the Dynamixel motors. It is an open source micro-controller based on 32-bit ARM cortex-M3 processor. A linear actuator control (LAC)

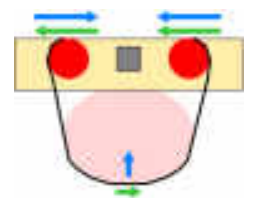

(a)

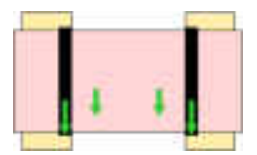

(c)

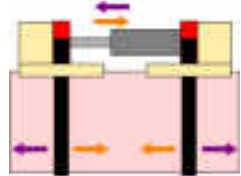

(b)

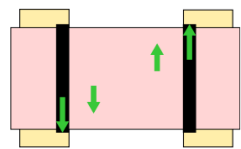

(d)
Fig. 3. Working principle of the hBracelet. (a) Squeezing (blue) and shear (green) forces due to the different pulleys spin direction. (b) Translational force (violet: opening; orange: closing) provided by the linear actuator. (c) Coherent shear force. (d) Opposite shear force.

board is used to control the linear actuator. It is a stand-alone closed-loop control board specifically designed for Actuonix "P" series actuators. Using the LAC allows us to greatly simplify the design and to reduce the development time, cost, and the processor overhead associated with direct motor control.

\section{Implementation}

The OpenCM9.04-C controller is connected with the computer and the Dynamixel motors through serial communication and TTL protocol, respectively. The LAC board receives a digital signal $\left(d_{L A C}\right)$ from the OpenCM9.04-C controller and in turn controls the motion of the linear actuator.

The linear actuator position $d_{\text {actuator }}$ is computed according to the duty cycle of the input signal $d_{L A C} \in[0,1]$ as

$$
d_{\text {actuator }}=d_{\text {LAC }} \text { Max } x_{\text {actuator }},
$$

where $M a x_{\text {actuator }}=2^{16}$.

Both the controller boards, the related electronic circuitry, and a battery pack are enclosed in a 3D printed box. While the linear actuator can be controlled only in position, the Dynamixel servo motors can be controlled both in velocity and position. In this application, we adopted velocity control for the "Auto-tuning procedure" (see Sec. II-D) and we took advantage of position control in all other cases to ensure better motion accuracy. The relationship between a single motor commanded angle $(\Delta \theta)$ and the corresponding belt length variation $(\Delta d)$ is

$$
\Delta d=r \Delta \theta
$$

where $r=12.5 \mathrm{~mm}$ is the radius of the servo motor pulley and $\Delta \theta$ is expressed in radians. From now onwards, we will refer to the movement of motors in terms of rotation angles.

\section{Auto-tuning procedure for the belts}

An auto-tuning procedure has been implemented to set the home position of the belts, in order to optimally pre-tension the system for best perception.

This is performed before any other action to make both belts in contact with the forearm. Indeed, at the beginning of each use the belts are fully open. The user wears the device 


\begin{tabular}{|c|c|c|c|c|c|c|c|}
\hline$T_{1}$ & $T_{2}$ & $T_{3}$ & $T_{4}$ & $T_{a}$ & Action & Sketch & Real effect \\
\hline 0 & 0 & 0 & 0 & 1 & Translation & & \\
\hline 1 & 1 & 0 & 0 & 0 & $\begin{array}{c}\text { Squeeze } \\
\text { (front) }\end{array}$ & & \\
\hline 0 & 0 & 1 & 1 & 0 & $\begin{array}{l}\text { Squeeze } \\
\text { (rear) }\end{array}$ & & \\
\hline 1 & 1 & 1 & 1 & 0 & $\begin{array}{c}\text { Squeeze } \\
\text { (whole arm) }\end{array}$ & & \\
\hline 1 & -1 & 0 & 0 & 0 & Shear (front) & & \\
\hline 0 & 0 & 1 & -1 & 0 & Shear (rear) & & \\
\hline 1 & -1 & 1 & -1 & 0 & $\begin{array}{c}\text { Shear } \\
\text { (coherent) }\end{array}$ & & \\
\hline 1 & -1 & -1 & 1 & 0 & $\begin{array}{c}\text { Shear } \\
\text { (opposite) }\end{array}$ & & \\
\hline
\end{tabular}

TABLE I

HAPTIC FEEDBACK MODALITIES THE HBRACELET CAN PROVIDE.

and the auto-tuning procedure starts. Motors, rotating in opposite direction inward at the same speed, move the belts up. The contact between the arm and the belt is recognized when the load of a motor among the two exceeds an a priori set threshold; the reached motor position is set as the home position of this belt.

\section{E. Working Principle}

Fig. 3 shows the four main types of haptic feedback the device can provide. In particular, when the pulleys of the motors which share the same belt spin in opposite directions (blue arrows), the belt applies a pressure on the user's arm generating a normal force (or release it, depending on the spin direction) as shown in Fig. 3a. When the motors spin in the same direction (green arrows), the belt applies a shear force to the skin. The linear actuator connecting the two bracelet parts is able to change their relative distance and therefore produce translational cues on the skin if no slippage occurs (see Fig. 3b). Since the hBracelet is equipped with two independently actuated belts, shear forces along different directions can generate different cutaneous sensations: when both the belts exert the tangential force along the same direction, the device provides a shear sensation, that might be either clockwise or counterclockwise (see Fig. 3c); when the two tangential forces have opposite direction the device

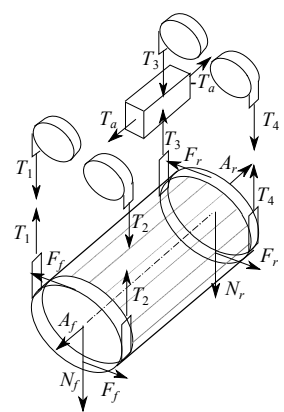

(a)

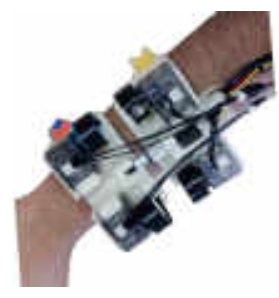

(b)
Fig. 4. (a) Scheme of the forces acting on the arm and on the hBracelet when motors are actuated. (b) A subject wearing the hBracelet.

provides a wringing effect, defined as opposite shear force in Fig. 3d.

Since each actuator of the hBracelet can be independently controlled, the squeezing action, or the shear force, can be provided by either one part of the device or both; the main $\mathrm{hBracelet}$ actuation types are summarized in Table I.

\section{FORCE ANALYSIS}

The scheme of the forces acting on the arm and on the $\mathrm{hBracelet}$ when the motors are actuated is shown in Fig. 4a, while Fig. $4 \mathrm{~b}$ shows a subject wearing the hBracelet on the forearm. Let us indicate with $T_{1}, T_{2}$ tension values on the front pulleys, with $T_{3}, T_{4}$ tension values on the rear ones, and with $T_{a}$ the linear actuator force.

The arm is subject to an overall action that can be represented as: i) a set of forces $A_{f}, A_{r}$ acting in the longitudinal direction of the arm ("translation"); ii) a set of forces $N_{f}, N_{r}$ acting on the normal (radial) direction of the arm ("squeeze"); iii) a set of tangential forces $F_{f}, F_{r}$ ("shear"). An action, intended as any combination between a force and a torque exerted on the arm, can be obtained by suitably controlling the belts' tension and the linear actuator force. In the longitudinal direction we easily get

$$
A_{f}=-A_{r}=T_{a}
$$

It is worth to underline that this expression is an approximation of the actual distribution of forces on the arm skin generated by the application of a force in the longitudinal direction. Indeed, such actions should also balance the bending moment due to the distance between actuator longitudinal main direction and arm longitudinal axis. However, in this application, since the structural force is filtered by the user's perception, we have assumed that the force in the longitudinal direction is dominant with respect to the distribution of tangential forces necessary to balance the bending moment, i.e., we have considered the longitudinal component of the force only. A more accurate estimation of the distribution of forces performed by the hBracelet is possible only considering also the compliance of arm tissues. Indeed, from the structural point of view the hBracelet fixed on the arm can be represented as an over-constrained structure; consequently, 
the complete equilibrium relationships can be solved only if the structural compliance of the system is known.

Considering the equilibrium of the $\mathrm{hBracelet}$ in the radial direction, the normal forces $N_{f}, N_{r}$ on the front and rear sections can be evaluated as

$$
N_{f}=T_{1}+T_{2}, \quad N_{r}=T_{3}+T_{4} .
$$

When $T_{1} \neq T_{2}$ and/or $T_{3} \neq T_{4}$ bracelet forces generate a torque on the arm that is balanced by tangential forces created by the friction on the contact surface. Assuming, for the sake of simplicity, that the cross section of the arm can be represented as a cylinder with a radius $R_{f}$ on the front section and $R_{r}$ on the rear one, the torques generated by the difference in the hBracelet tensions are given by

$$
\tau_{f}=R_{f}\left(T_{1}-T_{2}\right), \quad \tau_{r}=R_{r}\left(T_{3}-T_{4}\right)
$$

and the corresponding tangential forces on the arm surface are given by

$$
F_{f}=T_{1}-T_{2}, \quad F_{r}=T_{3}-T_{4} .
$$

Considering as a first approximation a linear relationship between the actuators force and forces exerted on the arm, we can summarize the above introduced relationships as follows

$$
\left[\begin{array}{c}
A_{f} \\
A_{r} \\
N_{f} \\
N_{r} \\
\tau_{f} \\
\tau_{r} \\
F_{f} \\
F_{r}
\end{array}\right]=\left[\begin{array}{ccccc}
0 & 0 & 0 & 0 & 1 \\
0 & 0 & 0 & 0 & -1 \\
1 & 1 & 0 & 0 & 0 \\
0 & 0 & 1 & 1 & 0 \\
R_{f} & -R_{f} & 0 & 0 & 0 \\
0 & 0 & R_{r} & -R_{r} & 0 \\
1 & -1 & 0 & 0 & 0 \\
0 & 0 & 1 & -1 & 0
\end{array}\right]\left[\begin{array}{c}
T_{1} \\
T_{2} \\
T_{3} \\
T_{4} \\
T_{a}
\end{array}\right]
$$

Such linear mapping can be used to generate composite actions on the arm, for example, if all the four actuators apply the same tension $T_{1}=T_{2}=T_{3}=T_{4}$, the same normal force is applied both on the front and rear sections of the arm, leading to a distributed normal force ("squeeze whole arm"). If tangential torques with the same direction are applied again on both the front and rear sections, a distributed tangential force is perceived by the user ("coherent shear"). If tangential torques have different signs, e.g., $\tau_{f}>0$ and $\tau_{r}<0$ or vice versa, a wringing force is applied to the arm ("opposite shear"). Some of these actions are schematically shown in Table I.

\section{HUMAN-ROBOT APPLICATIONS}

Human-Robot Interaction (HRI) is studied by researchers to understand and design robotic systems to use with or by humans. If the human and the robot are separated spatially or even temporally, we refer to these as remote interactions or teleoperation [?].

In order to demonstrate the effectiveness of the hBracelet, we conducted a qualitative experiment in a teleoperation scenario. The experiment had two main goals: (1) understanding

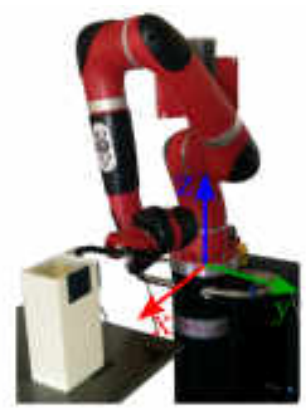

(a) Slave side

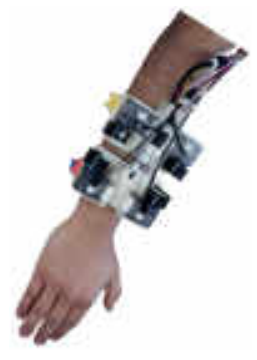

(b) Master side
Fig. 5. Experimental setup. (a) Sawyer manipulator with two force sensors mounted on the robotic gripper. (b) hBracelet able to provide cutaneous cues on a subject related to the information collected by the robot at a remote site.

how humans can be able to discern multiple haptic information provided on the same body area, i.e., the forearm, at the same time; (2) finding an intuitive mapping between the haptic stimuli the hBracelet can yield and some information coming from the remote environment.

a) Experimental setup: The proposed teleoperation system was composed of the hBracelet, detailed in Sec. II, a 7-DoF Sawyer manipulator (Rethink Robotics, US), and two OMD-20-SE-40N 3-DoF force sensors (Optoforce Ltd, $\mathrm{HU})$ mounted on the robotic gripper. The object was a parallelepiped with dimensions of $(80 \times 95 \times 20) \mathrm{mm}$ placed on a specific position of the table in front of the robot. Many small spheres of different size and weight were inserted into an opening on top of the object. The total weight of the object ranged from $160 \mathrm{~g}$ (empty object) to $600 \mathrm{~g}$ (with all the spheres inside) (see Fig. 5) .

b) Implementation: Using the control strategies detailed in Secs. II and III, information collected by the robot on the slave side was fed back to the user on the master side through the hBracelet. The exchange of messages between all the different devices was managed by the ROS framework, an opensource Robot Operating System [?]. ROS is not an operating system in the traditional sense of process management and scheduling; rather, it provides a structured communications layer above the host operating systems of a heterogeneous compute cluster. No real-time performance was required in this simplified teleoperation system: human subjects could command the motion of the slave robot using only few keys of keyboard, related to pre-determined motions of the robotic arm.

c) Participants: 10 right-handed subjects (7 males, 3 females, average age 26) participated in this qualitative study. Five of them had previous experience with haptic interfaces. None of the participants reported any deficiencies in their visual or haptic perception abilities. Participants were briefed about all the tasks and afterwards signed an informed consent, including the declaration of having no conflict of interest. All of them were able to give the consent autonomously. The participation in the experiment did not involve the processing of genetic information or personal data (e.g., health, sexual, lifestyle, ethnicity, political opinion, 


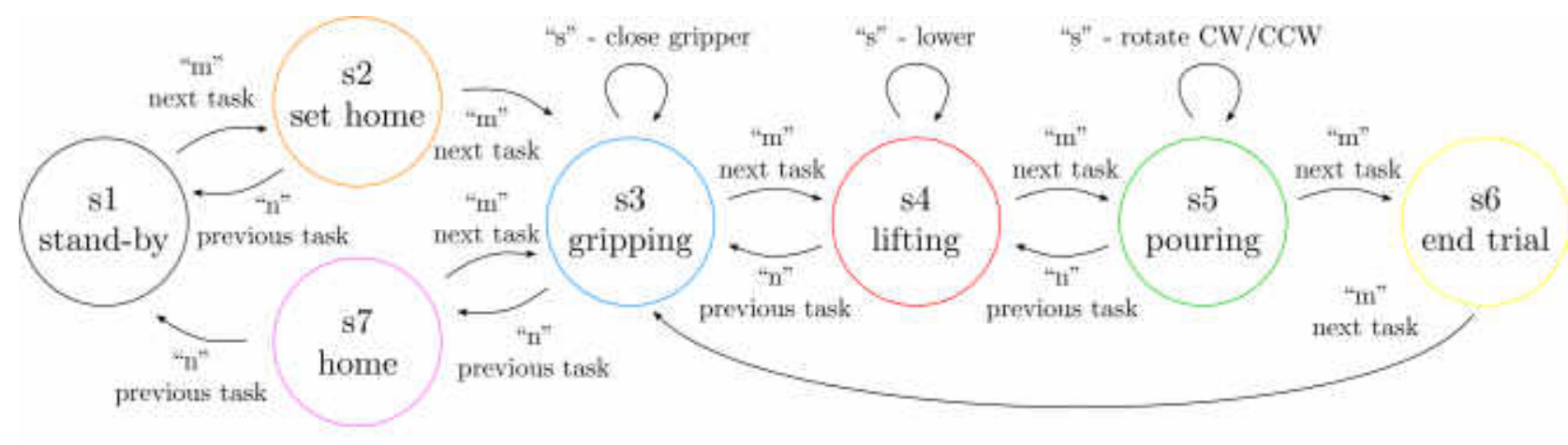

Fig. 6. The implemented finite state machine (FSM) to perform the experimental tasks.

religious or philosophical conviction). Our organization does not require any IRB review for this case.

d) Methods: Participants were asked to control the robot on the slave side by few keyboard inputs and without a direct visual feedback of the remote environment. All the possible behaviors of the robot are described by the finitestate machine shown in Fig. 6. Each arrow, i.e., system event, is characterized by a keyboard key. Because of the lack of visual feedback, the finite-state machine has been thought to be simple in terms of input commands: mainly, the operator uses " $m$ " and " $n$ " to change state and "s" to interact with the object. The type of interaction depends on the current state. Following all the states are described:

S1 - stand-by: the hBracelet and the Sawyer robotic arm are initialized; all the connections between each controller and the computer are established. The belts of the $\mathrm{hBracelet}$ are completely released to easily wear it and take it off;

S2 - set home: by means of the auto-tuning procedure that exploits motor torque readings (see Sec. II-D), the belts of the hBracelet come in contact with the forearm of the subject without compressing the skin; in the meanwhile the robotic arm reaches the starting position (see Fig. 7a);

S3 - gripping: the connection between the robot and the hBracelet is enabled, i.e., the motors on the haptic display move accordingly to what happens at the remote site; the robot end-effector approaches the object, that, at end of the motion, will be exactly between the gripper clamps (see Fig. 7b); subjects are asked to close the gripper until they feel a stable squeezing force, i.e., the gripper is exerting the required force on the object and will no longer close. Subjects command the progressive closing of the gripper by typing "s" (each time "s" is typed, the reference closing distance of the gripper decreases by $7 \mathrm{~mm}$ );

S4 - lifting: the closing distance, i.e., the closing force, of the gripper is kept constant. Subjects are asked to lift up the object until they feel a constant sensation of weight. Subjects command the progressive lifting of the object by typing "s" (each time "s" is typed the end-effector position increases by $1 \mathrm{~mm}$ upwards). Once the object is no longer in contact with the table (see Fig. 7c), the force along z-axis (see Fig. 5a) is constant, i.e., the sensation of the full object weight is perceived by the operator;

S5 - pouring: the robotic gripper automatically reaches a higher predetermined position (far enough from the table to do not hit it while rotating). This time, subjects are asked to rotate the object until they feel a change in its weight (see Fig. 7d). The preferred direction of pouring (randomly selected among clockwise $(\mathrm{CW})$ and counterclockwise (CCW) in each trial) is indicated to user via the hBracelet skin stretch action.

Subjects command the progressive rotation of the object by typing "s" (each time "s" is typed the object is rotated about the x-axis, as defined in Fig. $5 \mathrm{a}$, of $10^{\circ}$ ). When rotation reaches about $90^{\circ}$, the balls contained in the box begin to fall and the weight of the object changes;

S6 - end trial: when the subjects feel the change in weight, they can move to the last state completing the trial. A message appears on the screen in front of the subject to confirm the end of the trial, then by pressing " $\mathrm{m}$ " again, the object is placed back on the table;

S7 - home: this state is reached only when we go back from the gripping state by typing "n." The robotic arm goes back to its starting position without setting a new home for the hBracelet, i.e., without repeating the auto-tuning procedure.

An experimental trial can be considered accomplished when, starting from S1, S6 ("end trial") is visited.

Since subjects do not see what is happening at the remote site, each transition between subsequent states strongly depends on what subjects perceive on their forearm by means of the proposed haptic interface. Headphones were worn by subjects to mask the noise of the motors. During this experimental evaluation we mapped three different pieces of information concerning the robot's state to three different haptic stimuli:

- average gripping force sensed by the two force sensors mounted on the gripper $\rightarrow$ normal force of the hBracelet ("squeeze");

- weight of the object (force along the z-axis in Fig. 5a) $\rightarrow$ longitudinal stretch/compression of the skin exerted through the linear actuator of the hBracelet ("transla- 


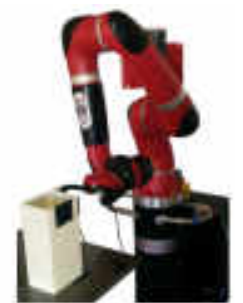

(a) home

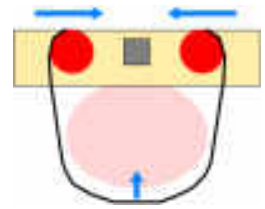

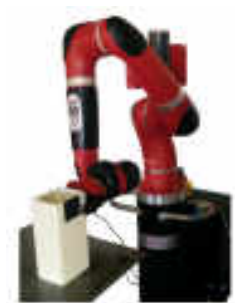

(b) gripping

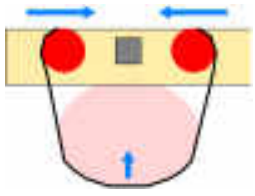

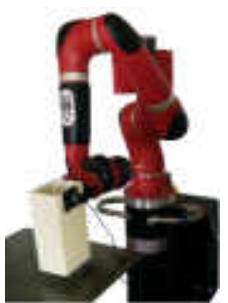

(c) lifting

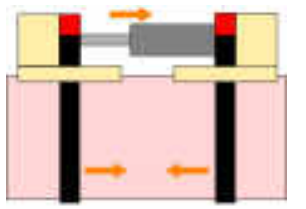

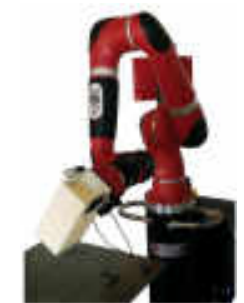

(d) pouring

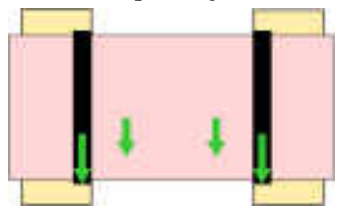

Fig. 7. Sawyer manipulator positions (top) and corresponding hBracelet actions (bottom) during the main experimental tasks. The hBracelet actions refer only to the haptic feedback combination numbered as 1 in Table II as an example of use.

tion");

- torque about the $\mathrm{x}$-axis (as defined in Fig. 5) estimated on the end-effector $\rightarrow$ tangential or wringing action of the hBracelet ("shear").

We decided to use this specific mapping strategy because, in our opinion, this way the actions of the hBracelet recall as much as possible the real interaction with the remote object and subjects could intuitively understand what was happening at the remote site.

Besides the general mapping strategy detailed above, both the longitudinal motion of the linear actuator and the shear action can have some variants. Specifically, we can choose to set the initial position of the linear actuator either fully extended, or fully retracted. Similarly, we can choose to provide to the subject the sensation of torque using either the action defined as "shear opposite," or the one defined as "shear coherent." During this experimental evaluation the four combinations shown in Table II have been tested by each participant.

The intensity of all haptic stimuli was proportional to the measures gathered on the slave side. The force exerted by the robot during the grasping of the object ranged from $0 \mathrm{~N}$ to $10 \mathrm{~N}$. In order to maximize the perception of the squeezing action, the force range has been mapped on the hBracelet motors rotation from $0^{\circ}$ to $60^{\circ}$; the force exerted by the robot to hold the object (along the z-axis in Fig. 5a) varied from $0 \mathrm{~N}$ to $15 \mathrm{~N}$, and it has been mapped on the range $8000-45000$ to command the linear actuator; the torque estimated by the robot ranged from $-1 \mathrm{Nm}$ and $1 \mathrm{Nm}$ and has been mapped on the hBracelet motors rotation from $-80^{\circ}$ to $80^{\circ}$ regarding the shear action. All the rotation angles are measured with respect to the motors home position set during the auto-tuning procedure detailed in Sec. II-D.

e) Evaluation: After completing the test, the participants were asked to fill out a questionnaire which sought to evaluate the value of transmitting robot state information to the user via the hBracelet. In the first part subjects had to provide their demographic information, such as age and gender; in the second part they had to evaluate each feedback

\begin{tabular}{|c|c|c|c|}
\hline & object weight & gripping force & torque \\
\hline 1 & translation inward & squeeze & shear coherent \\
\hline 2 & translation outward & squeeze & shear coherent \\
\hline 3 & translation inward & squeeze & shear opposite \\
\hline 4 & translation outward & squeeze & shear opposite \\
\hline
\end{tabular}

TABLE II

THE TABLE SHOWS HOW TASK FEATURES ARE MAPPED TO THE DEVICE.

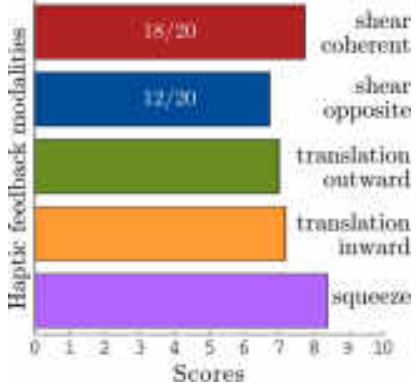

(a)

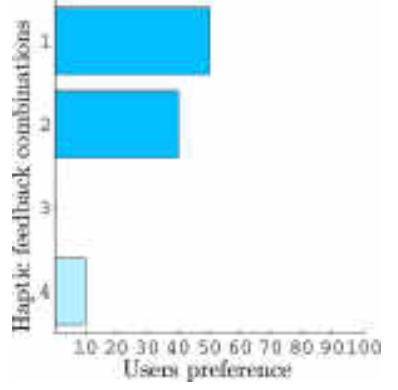

(b)
Fig. 8. Questionnaire replies. (a) Means of evaluation of each feedback modality (according to a 10-point scale). On the top two bars the number of times users guessed the direction of rotation of the grasped object using that feedback modality. (b) Subjects preference (\%) about the best haptic combination provided by the hBracelet among the four listed in Table II

modality according to a 10 -point scale, in particular subjects had to choose a maximum score (10) if they completely agreed and a minimum score (1) if they completely disagreed.

\section{RESULTS AND DISCUSSION}

Figure 8a shows the mean of the evaluations given by the participants for each feedback modality in order to understand which was the preferred modality for each robot state, i.e., the haptic signals that provided the clearest image of what was happening on the slave side. Apart the evaluation of each feedback modality, i.e., "shear opposite," "shear 
coherent," "translation outward," "translation inward," and "squeeze," in the last question, participants were asked to choose the best haptic combination among the ones summarized in Table II, that gave them the feeling of performing better throughout the experiment. Subjects preference is reported in Fig. $8 \mathrm{~b}$ in which on the y-axis haptic feedback combinations, numbered as in Table II, are shown, while the $\mathrm{x}$-axis represents the percentage of people who preferred that specific combination compared to the others. A single response was accepted for each user.

As we can see from the Figure $8 \mathrm{~b}$ :

- $50 \%$ of subjects chose the combination of "translation inward," "squeeze" and "shear coherent";

- $40 \%$ of subjects chose the combination of "translation outward," "squeeze" and "shear coherent";

- $10 \%$ of subjects chose the combination of "translation outward", "squeeze" and "shear opposite".

The results obtained show that the squeeze action exerts a clear stimulus, useful in informing the subject about the force the object is grasped with. Furthermore, there seems to be no difference between the two modalities of translation, in fact they have reached a very similar number of preferences. The most interesting result concerns the shear action; in particular $90 \%$ of participants preferred "shear coherent" instead of "shear opposite." This result was not as evident in Fig. 8a, even though the score for the "shear coherent" was more than 1 point higher. It is worth pointing out that the use of the "shear coherent" modality led to a significantly better understanding of the rotation direction of the objects. As shown on the top two bars of Fig. 8a, the number of times the users guessed the direction of rotation of the grasped object is 18 for the "shear coherent" and 12 for the "shear opposite," over a total of 20 trials. The total number of trials is 20 because the "shear coherent" action was present in two feedback combinations, the first and the second (see Table II). The same for the "shear opposite" actions that was present in both the third and fourth combinations.

\section{CONCLUSION AND FUTURE WORK}

This work presents a novel wearable force feedback haptic device for the upper limb which we called "hBracelet." It is able to provide the distributed mechanotactile stimulation on the user arm skin by means of pressure and stretch cues related to normal, tangential, and longitudinal forces. We described the mechanical structure, working principle, mathematical formulation, and control of the proposed device.

In order to evaluate the performance of the hBracelet, we conducted a qualitative experiment in a teleoperation scenario where the hBracelet was worn by a human and a robot was in remote communication via the device. This experiment allowed us to characterize the haptic feedback of the developed device. Furthermore, it provided us some information to understand how humans can be able to discern multiple haptic cues provided on the same body area, i.e., the forearm, at the same time and also to find an intuitive mapping between the haptic stimuli the hBracelet can yield and some information coming from a remote environment.
The results show that the device is capable of informing about the forces acting at the remote site while performing a grasping and pouring task, hence improving the performance of the teleoperation system. In the future, we plan to run a more extensive evaluation, enrolling more human subjects and rigorously assessing the value of distributed mechanotactile stimulation. Moreover, we aim to improve the wearabiltiy and ergonomics of the device by reducing the number of actuators, or selecting more compact form-factor ones.

Finally, a comparison evaluation with a device capable of providing direction cues by means of vibrotactile patterns is of interest. We are also considering the possibility of integrating one or more vibrotactile motors in the new design of our haptic interface. 